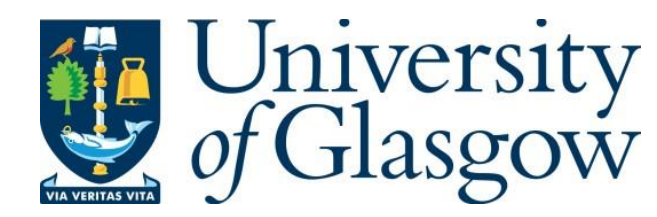

Yang, S., Lemke, C., Cox, B. F., Newton, I. P., Cochran, S. and Nathke, I. (2020) Deep Compressed Sensing for Characterizing Inflammation Severity with Microultrasound. In: 2020 IEEE International Ultrasonics Symposium (IUS), 07-11 Sep 2020, ISBN 9781728154480 .

There may be differences between this version and the published version. You are advised to consult the publisher's version if you wish to cite from it.

http://eprints.gla.ac.uk/231087/

Deposited on: 3 September 2021

Enlighten - Research publications by members of the University of Glasgow http://eprints.gla.ac.uk 


\section{Deep Compressed Sensing for Characterizing Inflammation Severity with Microultrasound}

\author{
Shufan Yang \\ James Watt School of Engineering \\ University of Glasgow \\ Glasgow, United Kingdom \\ https://orcid.org/0000-0003-0531-2903
}

\author{
Christina Lemke \\ James Watt School of Engineering \\ University of Glasgow \\ Glasgow, United Kingdom \\ https://orcid.org/0000-0003-4003-4814
}

\author{
Ben F. Cox \\ School of Medicine \\ University of Dundee \\ Dundee, United Kingdom \\ b.cox@dundee.ac.uk
}

\author{
Ian P. Newton \\ School of Life Sciences \\ University of Dundee \\ Dundee, United Kingdom \\ i.z.newton@dundee.ac.uk
}

\author{
Sandy Cochran \\ James Watt School of Engineering \\ University of Glasgow \\ Glasgow, United Kingdom \\ https://orcid.org/0000-0001-7324-7790
}

\author{
Inke Näthke \\ School of Life Sciences \\ University of Dundee \\ Dundee, United Kingdom \\ https://orcid.org/0000-0003-2420-4385
}

\begin{abstract}
With histological information on inflammation status as the ground truth, deep learning methods can be used as a classifier to distinguish different stages of bowel inflammation based on microultrasound ( $\mu \mathrm{US}$ ) B-scan images. However, it is extremely time consuming and animal usage is high to obtain a balanced data set for every stage of inflammation. In this study, we describe a deep compressed sensing method to increase the number of B-scan images for inflammation studies without use of additional animals. In this way, training data can be quickly augmented. The fidelity of the synthesized data is evaluated using both qualitative and quantitative methods. We find that the synthetic data have high structural similarity when compared with original B-scan images. Further evaluation, such as finding the correlation of $\mu \mathrm{US}$ and microscopy images and calculating attenuation coefficient, will be investigated in future to provide better understanding.

Index Terms-B-scan images, Deep Learning, Generative Ad-
\end{abstract} versarial Network (GAN), Microultrasound.

\section{INTRODUCTION}

Different levels of acute inflammation of the gastrointestinal tract tissue can be found during the active phases of Crohn's disease [1] [2]. Although deep learning methods have the potential to classify this inflammation, an insufficient number of B-scan image training data prevents adaption of these methods to form clinical practice guidelines. Moreover, it is extremely time consuming and inimical with animal welfare to obtain a balanced dataset with all inflammation stages directly from mice. This is because of the time required to treat the mice, sacrifice them and dissect the bowel tissue for scanning. Learning from widely used image translation in autonomous driving scenarios, recent developments in artificial neural networks allow researchers to generate synthetic images using the image translation method [3].

In general, training images can be simply rotated, re-scaled or flipped to create additional images and expand the size of the training set. However, these modifications introduce only minor additional diversity and the new images are very likely to be correlated with the original images. Inspired by the image-to-image translation research [4], we developed a deep sensing method based on generative adversarial networks (GANs) [4], built on the open access TensorFlow 2.0 platform [5].

GANs and their variations have recently become popular in image applications involving multi-sensor data fusion to generate synthetic images. For instance, a deep convolutional GAN framework was used to supplement a data set of computed tomography images of liver lesions [6]. With the synthetic data, the author demonstrated a neural networkbased classification with three classes. Another example is of positron emission tomography images synthesised from CT images [7]. However, to the best of our knowledge, the use of a GAN to generate synthetic microultrasound $(>30 \mathrm{MHz})$ B-scan images has not previously been considered.

\section{METHOD}

\section{A. Inflammatory Grading}

An inflammatory grading scheme for mouse bowel was adapted from Elsheikh et al. and Erben et al. [8] [9]. As shown in Fig. 1, Grade 0 corresponds with lack of signs of inflammatory cell infiltration and the presence of a continuous, intact epithelium. Grade 1 corresponds with cases where there are signs of mucosal inflammatory cell infiltration (red arrows) but no mucosal disruption. Grade 2 corresponds with cases of cell infiltration at the mucosa and submucosa (green arrows) and focal epithelial disruption (blue arrow). Finally, Grade 3 corresponds with cases such as the one shown, with transmural inflammation and cell infiltration at all histologic levels and confluent disruption of the epithelium. The data 


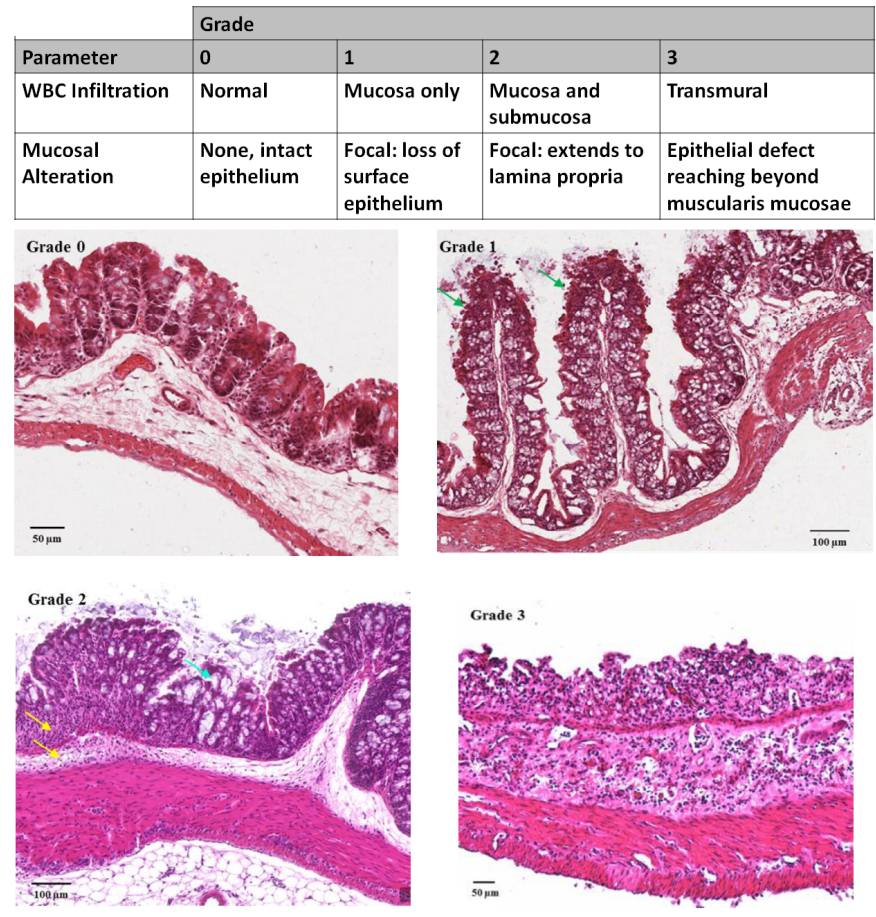

Fig. 1. Inflammation severity using histology information of mouse bowel samples. The top table shows criteria for individual grades of inflammation severity. Key: WBC-White blood cells.

set we collected for this study has a limited sample size for Grade 1 and Grade 2 [10]. The means to generate sufficient $\mathrm{B}$-scan training images is a challenging task.

\section{B. The Deep Compressed Sensing Method}

Various forms of generating synthetic images can be cast as image transformation problems where the input image from one domain is mapped to an output image from another domain. Numerous methods have been proposed recently to perform image transformation tasks as supervised regression. As shown in Fig. 2, a set of randomly selected unprocessed scan line data allows the discriminator network to self-adjust and generate a synthetic image. Based on game theory, the GAN will finally generate a good synthetic image which has characteristics as close as possible to the real image. The initial goal inherited from a GAN is to learn the mapping function between the two domains: randomly selected scan line data and B-mode images.

The pseudo code for deep compressed sensing is shown below in Fig. 3.

The generator, $G$, uses the original environment input, $o$, and noise, $n$, to generate the synthetic image, $p$. In other words, $G:\{o, n\} \longrightarrow p$. The adversarial discriminator, $D$, tries to classify the input, $o$ concatenating with $p$ and the input $o$ concatenating with the real B-scan image, $r$.
$\mathcal{L}_{\mathrm{GAN}}(G, D)=\mathbb{E}_{o, p}[\log D(o, p)]+\mathbb{E}_{o, n}[\log (1-D(o, G(o, n))]$

As shown in Equation 1, $G$ aims to minimize the objective, $\mathcal{L}_{\mathrm{GAN}}$, and $D$ tries to maximize the objective, $\mathcal{L}_{\mathrm{GAN}}$.

$$
\mathcal{L}_{L 1}(G)=\mathbb{E}_{o, p, n}\left[\|p-G(o, n)\|_{2}\right]
$$

The generator objective for optimizing divergence between the generated data and input real data is:

$$
G_{\text {global }}=\arg \min _{G} \max _{D} \mathcal{L}_{G A N}(G, D)+\lambda \mathcal{L}_{L 1}(G)
$$

In the work reported here, the data used for learning were obtained with a customized $\mu \mathrm{US}$ platform [10]. After comparison with histology ground truth, the B-scan image inflammation level is received as the label. In our work, the generator consists of a u-net network and the discriminator network consists of four fully connected convolution layers with 64 hidden units followed by a LeakyReLU activation function, except for the final output layer. The dimension of latent noise is 16 . Both networks were trained with the RMSProp optimizer with a learning rate of 0.001 . We trained the model for 10,000 iterations with different regularization coefficients. The least square loss function suggested in [11] was used in all the experiments.

\section{RESULTS AND DISCUSSION}

Compressed sensing was applied to create sparse representations of the spatial and temporal information of $\mu \mathrm{US}$ signals in radio frequency (RF) form. RF data were obtained from $N_{\text {DSS }}$ $=35$ mice treated with dextran sodium sulfate (DSS) via their drinking water ad libitum, and $N_{\mathrm{CON}}=12$ untreated mice. Ex vivo samples of the small bowel, caecum and colon were resected and scanned with a $>35 \mathrm{MHz}$ single-element focused piezocomposite transducer. The grade of inflammation was determined by haematoxylin and eosin (H\&E) histology as the ground truth. The resulting sparse measurement metrics were used to train a GAN to create new metrics [4], from which we reconstructed RF data as the basis for B-scan images.

\section{A. Qualitative Results}

We show in Fig. 4 that the proposed method can produce new B-scan images based on RF signals, here from ex vivo mouse bowel tissue at mild-moderate-severe levels of inflammation. The proposed approach successfully generated B-scan images that showed the layers of mouse bowel tissue and representative tissue shapes corresponding with histology observations.

Fig. 5. (top row) shows that GAN G can generate highquality synthetic B-mode images. The inputs of the algorithm are ground-truth (bottom row). The generator network generated the synthetic images using randomly-selected scaled scan-line images. The generated synthetic images have the same correlation with real B-scan images, although with some inconsistencies such as is visible in Fig. 5(a). 


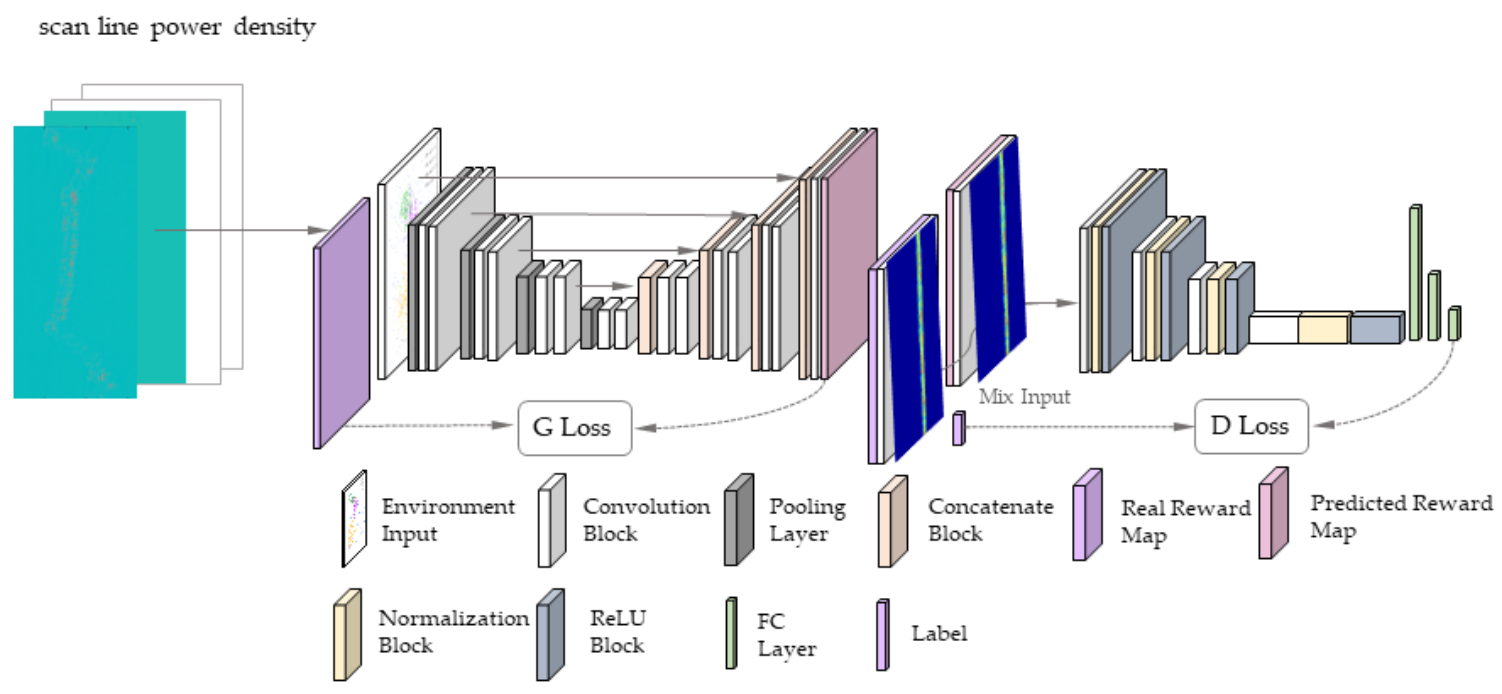

Fig. 2. Deep compressed sensing network.

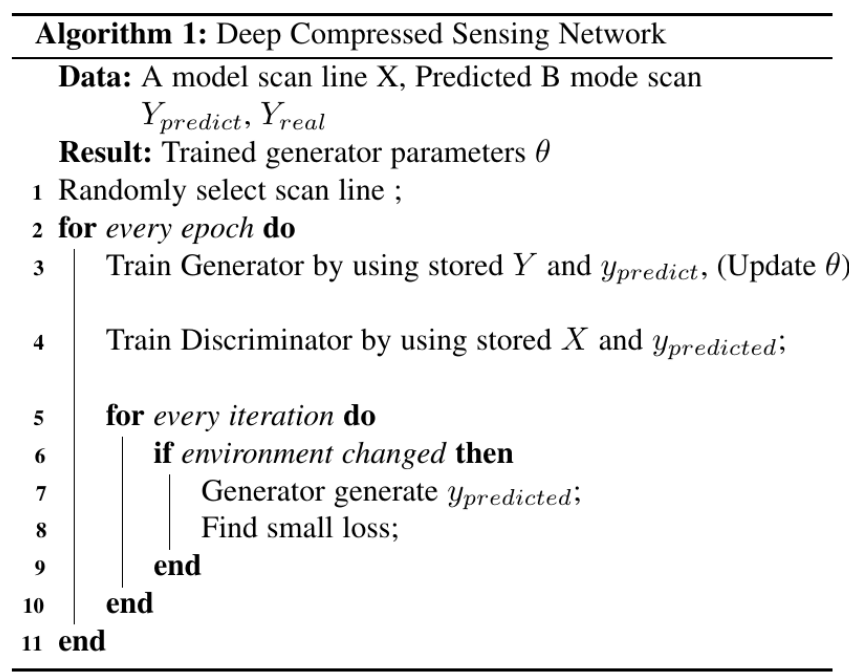

Fig. 3. Pseudo code for the deep compressed sensing network.

\section{B. Quantitative Results}

For evaluation of the quality of the images generated by our method, we used a structured similarity index, SSIM [12] to check the quality of the synthetic images. The following equations show how the SSIM works:

$$
\begin{gathered}
L_{x, y}=\frac{2 u_{x} u_{y}+C_{1}}{u_{x}^{2}+u_{y}^{2}+C_{1}} \\
C_{x, y}=\frac{2 \sigma_{x y}+C_{2}}{\sigma_{x}^{2}+\sigma_{y}^{2}+C_{2}} \\
S_{x, y}=\frac{\sigma_{x y}+C_{3}}{\sigma_{x} \sigma_{y}+C_{3}}
\end{gathered}
$$

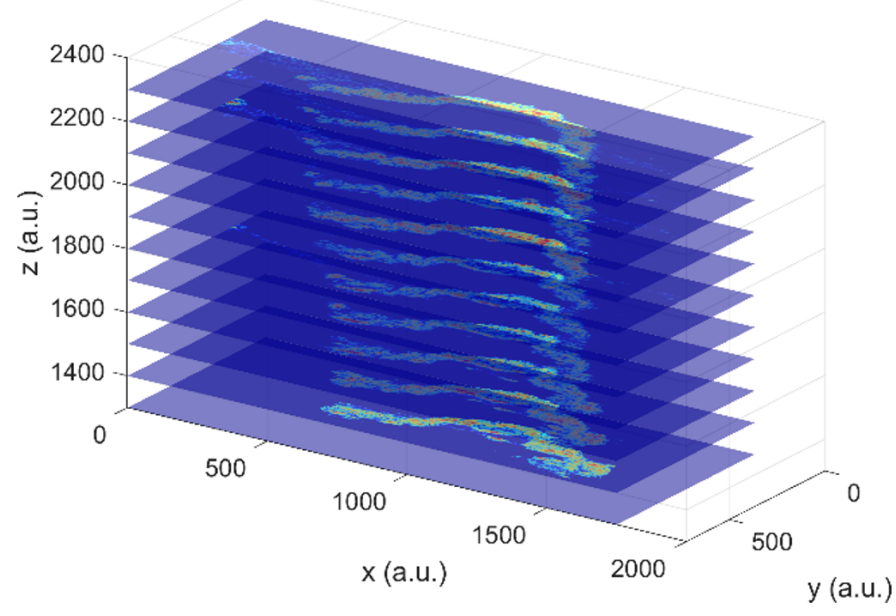

Fig. 4. Representation of generated B-scans of mouse bowel tissue (caecum) and inflammation features consistent with histological observations. The $\mathrm{x}, \mathrm{y}$, $\mathrm{z}$ axes correspond to the image size.

where $u_{x}, u_{y}$ represent the mean values of the image $\mathrm{X}$ and $\mathrm{Y} ; \sigma_{x}, \sigma_{y}$ represent the standard deviation of the image $\mathrm{X}$ and $\mathrm{Y} ; \sigma_{x}^{2}, \sigma_{y}^{2}$ represent the variance and $\sigma_{x y}$ represents the covariance. $C_{1}, C_{2}$, and $C_{3}$ are constants applied to keep the denominator from being zero. In our experiment, $C_{1}=\left(K_{1} \cdot L\right)^{2} ; C_{2}=\left(K_{2} \cdot L\right)^{2} ; C_{3}=C_{2} / 2 ; K_{1}=0.01$, $K_{2}=0.03, L=255 . L$ is the dynamic range of pixel values, generally set to 256 and $K_{1} \ll 1, K_{2} \ll 1$ are scalar constants [12]. Finally, the SSIM is:

$$
\operatorname{SSIM}(X, Y)=L(X, Y) \cdot C(X, Y) \cdot S(X, Y)
$$

When the setting of the $C_{3}$ is equal to $C_{2} / 2$, then Eq. 7 


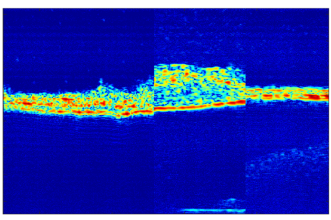

(a)

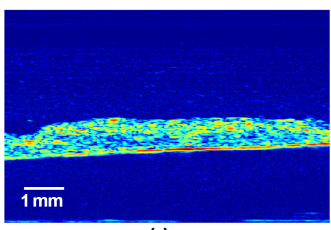

(e)

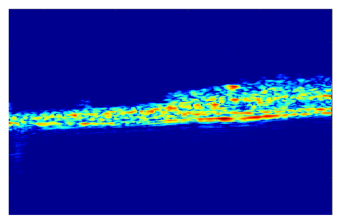

(b)

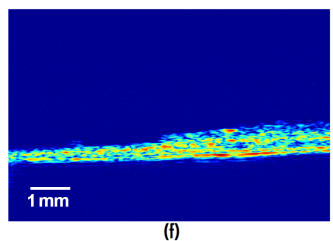

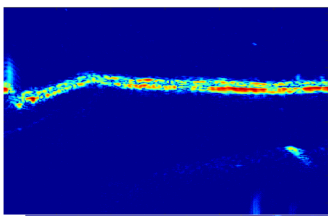

(c)

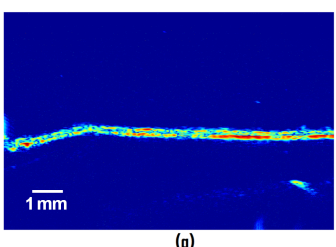

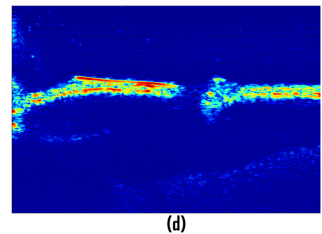

(d)

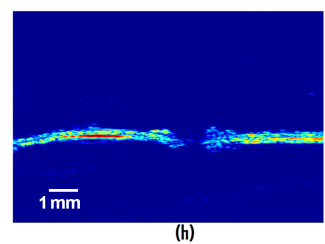

Fig. 5. Synthetic image results (top) paired with the ground truth B-Scan images of caecum samples (bottom) for comparison.

can be simplified to Eq. 8 .

$$
\operatorname{SSIM}(X, Y)=\frac{\left(2 u_{x} u_{y}+C_{1}\right)\left(2 \sigma_{x y}+C_{2}\right)}{\left(u_{x}^{2}+u_{y}^{2}+C_{1}\right)\left(\sigma_{x}^{2}+\sigma_{y}^{2}+C_{2}\right)}
$$

TABLE I

COMPARISON OF SYNTHETIC B-SCAN IMAGE AND SAMPLE OF GRADE 3 AND GRADE 0 INFLAMMATION

\begin{tabular}{|c|c|c|c|c|}
\hline & $(\mathrm{a})(\mathrm{e})$ & $(\mathrm{b})(\mathrm{f})$ & $(\mathrm{c})(\mathrm{g})$ & $(\mathrm{d})(\mathrm{h})$ \\
\hline SSIM & 0.7446 & 0.7131 & 0.7026 & 0.7411 \\
\hline
\end{tabular}

As shown in Fig.5, (a) and (b) are synthetic B-scan images for Grade 3 inflammation samples. (e) and (f) are samples of original B-scans for Grade 3 inflammation. (c) and (d) are synthetic B-scan images for Grade 0 inflammation samples. Finally (g) and (h) are original B-scans for Grade 0 . The value of the original (real) B-scan images and the synthetic B-scan images are consistent, with values around 0.7. However, we appreciate that this single indication is insufficient for final evaluation and further studies are needed to investigate the technique in more detail.

\section{Conclusion}

This paper describes our development of a deep compressed sensing network based on a GAN to generate synthetic Bmode microultrasound images. Using this advanced generative learning method, we increased the number of available training data sets of microultrasound B-scan images based on mouse bowel scans with different levels of inflammation. We used the SSIM method to compare the real B-scan samples and synthesized data with results that suggest further work will be worthwhile. The next step is to evaluate the validity of the synthetic data further based on its lateral resolution.

\section{ACKNOWLEDGMENTS}

The mouse bowel microultrasound data collection was supported by the UK Engineering and Physical Sciences Research Council (EPSRC) grant number EP/K034537/2, "Sonopill" and was conducted under a Home Office (UK) Procedure Project Licence: P3800598E, in accordance with the Animal (Scientific Procedures) Act 1986.

\section{REFERENCES}

[1] B. F. Cox, F. Stewart, H. Lay, G. Cummins, I. P. Newton, M. P. Desmulliez, R.J. Steele, I. Näthke, and S. Cochran, "Ultrasound capsule endoscopy: sounding out the future," Ann. Transl. Med., vol. 5, no. 9, pp. 201-201, May 2017.

[2] G. Cummins et al., Gastrointestinal diagnosis using non-white light imaging capsule endoscopy, Nat. Rev. Gastroenterol. Hepatol., 2019.

[3] L. Wang, W. Cho, and K.J. Yoon, Deceiving image-to-image translation networks for autonomous driving with adversarial perturbations. IEEE Robotics and Automation Letters, 5(2), 1421-1428, 2020.

[4] I. Goodfellow et al., Generative Adversarial Nets, Adv. in Neural Info. Proc. Systems, 2014

[5] P. Singh and A. Manure, Introduction to TensorFlow 2.0. In Learn TensorFlow 2.0 (pp. 1-24). Apress, Berkeley, CA, 2020.

[6] M. Frid-Adar, E. Klang, M. Amitai, J. Goldberger, and H. Greenspan, Synthetic Data Augmentation using GAN for Improved Liver Lesion Classification. arXiv 2018, arXiv:1801.02385.

[7] A. Ben-Cohen, E. Klang, S.P. Raskin, S. Soffer, S. Ben-Haim, E. Konen, M.M. Amitai and H. Greenspan, Cross-Modality Synthesis from CT to PET using FCN and GAN Networks for Improved Automated Lesion Detection. Eng. Appl. Artif. Intell. 2019, 78, 186-194.

[8] W. Elsheikh, K. L. Flannigan, W. McKnight, J. G. P. Ferraz, and J. L. Wallace, "Dextran sulfate sodium induces pan-gastroenteritis in rodents: implications for studies of colitis.," J. Physiol. Pharmacol., vol. 63, no. 5, pp. 463-469, Oct. 2012.

[9] U. Erben et al., "A guide to histomorphological evaluation of intestinal inflammation in mouse models," Int. J. Clin. Exp. Pathol., vol. 7, no. 8, pp. 4557-4576, 2014.

[10] S. Yang, C. Lemke, B. F. Cox, I. P. Newton, I. Näthke and S Cochran, "A Learning Based Microultrasound System for the Detection of Inflammation of the Gastrointestinal Tract," in IEEE Transactions on Medical Imaging, doi: 10.1109/TMI.2020.3021560, 2020.

[11] G. E. Hinton, S. Osindero, and Y.-W. Teh, "A fast learning algorithm for deep belief nets," Neural Comput., vol. 18, no. 7, pp. 1527-1554, 2006.

[12] M-J. Chen , A. C. Bovik, "Fast structural similarity index algorithm," J Real-Time Image Proc, 6:281-287, 2011. 